\title{
Optimised Filters for Texture Defect Detection
}

\author{
J. L. Sobral \\ Departamento de Informática \\ Universidade do Minho \\ 4700 - 057 Braga, PORTUGAL
}

\begin{abstract}
This paper presents a new approach to texture defect detection based on a set of optimised filters. Each filter is applied to one wavelet sub-band and its size and shape are tuned for a defect type. The wavelet transform provides a very efficient way to decompose a complex texture into a set of base components (wavelet sub-bands), which are then analysed by each filter to detect a kind of defect. The proposed methodology has been successfully applied to leather inspection, achieving the detection rate of highly trained human operators. The process is also fast enough to be used for in-line inspection.
\end{abstract}

Keywords-wavelet, defect detection, leather inspection

\section{INTRODUCTION}

Natural textures, such as sand, marble, granite and leather are very difficult to analyse, since texture features present a high variation, even among a single texture sample. Usually, in these texture types, texture features follow a probability distribution and cannot be conveniently characterised through first and second order statistics.

Texture analysis through filter bank approaches [1] is based on a set of convolution masks. Each mask acts as a filter that responds to a particular frequency, producing a texture feature. The result from several filters can be used for texture classification, since different textures may have different responses on each frequency. These approaches can be interpreted as a partition of the texture power spectrum, where each filter is tuned for a particular frequency with a predefined bandwidth and orientation.

One of the most used approaches for texture analysis is based on Gabor filters [2], due to its optimal join spatial and frequency localisation. In this approach, texture features are calculated based on a set of Gabor filters, performing a nearly uniform (dyadic) coverage of the frequency domain.

Defect detection on textured materials usually requires more specific filters, since changes in the power spectrum can be very small, which may not be detected by generic filter masks. Several authors have addressed the design of optimised Gabor filters [3][4]. The main idea is to tune each Gabor filter for defect type. A Gabor filter has several parameters, namely the filter central frequency, the filter bandwidth among $X$ and $\mathrm{Y}$ direction and the filter orientation. Some restrictions to these parameters should be introduced, since an exhaustive search in the solution space is not feasible.

This work was partially supported by project "SIGMA - Sistema de Geo-referenciação Móvel assistido por Imagem", funded by "AdI - Agência de Inovação", POSI.
Gabor filters have several limitations, mainly due to their high computing costs. First, they generate a complex convolution mask, which requires two convolutions per filter. Second, masks are not separable and should have a medium size to perform an accurate power spectrum partition. Third, the amount of data increases with the same ratio as the number of the filters, since each filter output has the same size as the original image. The strong point of the Gabor filters is that they can perform a very flexible power spectrum partition.

The wavelet decomposition [5] overcomes most of these performance limitations. It uses a set of real value 1D masks to perform a recursive power spectrum partition, down sampling the image after each filtering step, which keeps the data set constant. Wavelet decomposition imposes a predefined power spectrum partition, originating a logarithmic decomposition that performs a finer partition in the lower frequency space. This limitation is partially overcome by wavelet packets [6], which recursively decompose both low and high frequencies. Intermediate frequencies are particularly important in textured materials, since these frequencies usually carry most of the texture information.

The next section describes the proposed optimised filters, which are based on the wavelet sub-band decomposition. The third section presents the methodology to obtain the optimised filters. The forth section presents performance results and the last section discuss these results and future work.

\section{OPTIMISED FILTERS}

Defect detection in textured materials can be very subjective, since defects can be very subtle. Defects can be highly localised in space, which may lead to a strong modification in a wide range of the power spectrum, but they can also be less localised in space, which may lead to a small modification in the power spectrum. Therefore, changes in the power spectrum should be detected either by highly frequency localised filters, or by less frequency localised filters, depending on the defect to be detected. The filter size also affects the defect detection success: less space localised defects usually require larger filters.

The wavelet transform provides an excellent framework to deal with multi-scale defects (both in space and frequency). The first level of the wavelet packet decomposition divides the power spectrum into four square parts; the second level divides the same spectrum in sixteen square parts and the third in sixty-four parts. A filter applied in the first level of the wavelet covers one fourth of the entire spectrum, while one applied in the second level only covers one sixteen of the spectrum. 
Therefore, filters applied in higher wavelet decomposition levels are more localised in the frequency domain and are tuned to detect defects that are easier to detect by analysing only a small part of the spectrum. Conversely, filters applied in the first levels can detect broader spectrum changes.

Defect size and orientation can increase the complexity of the optimised filter design, particularly when defects are very subtle. Traditional approaches are based on manual image segmentation to identify areas of possible defects that are later used to obtain optimised filters. This process relies on accurate image segmentation since defect features are calculated over the entire defect area. To overcome this limitation the proposed optimised filters are calculated over a small area inside the defect that minimises or maximises the feature value. The minimum or maximum value calculated on that small area is used to represent the entire defect area. This approach removes the burden of accurate segmentation, since it requires only that the defect should be included in the segmented area. Also the approach allows the optimised filter selection process to search for the most relevant feature for each defect type.

Each optimised filter calculates the average energy in a wavelet sub-band (i.e., on a specific part of the power spectrum), with a window size and orientation tuned for a type of defect. The energy is calculated by using two alternatives: the average value and the standard deviation.

Overall the defect detection process first performs the wavelet transform and then applies a bank of filters to calculate the average energy on several frequency channels. Each filter has a predefined window size and orientation. The output of each filter might indicate the presence of a defect region, which can be a high or low value, depending of the filter.

Initially, the image is segmented into a set of regions and only that set of regions is analysed to speed up the filter calculation. These regions correspond to areas of high/low intensity and contrast, which should include defect regions, as well as a large amount of normal regions. The calculated features for each filter are normalised to decrease the sensibility among different samples.

\section{FiLTER SELECTION}

The filter selection process chooses the most appropriate wavelet sub-band, filter size and orientation for each defect type. In a training phase, a large number of predefined smoothing filters are applied to each wavelet sub-band and, afterwards, the filters that can detect more defects are selected.

The first step applies the wavelet packet decomposition to each image with three levels (Figure 1). This figure also exemplifies the numbering used for each wavelet sub-band.

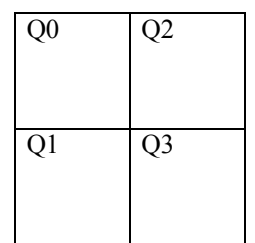

\begin{tabular}{|l|l|l|l|}
\hline Q4 & Q6 & Q12 & Q14 \\
\hline Q5 & Q7 & Q13 & Q15 \\
\hline Q8 & Q10 & Q16 & Q18 \\
\hline Q9 & Q11 & Q17 & Q19 \\
\hline
\end{tabular}

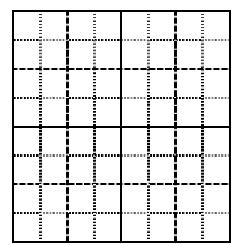

Figure 1 - Wavelet packet decomposition

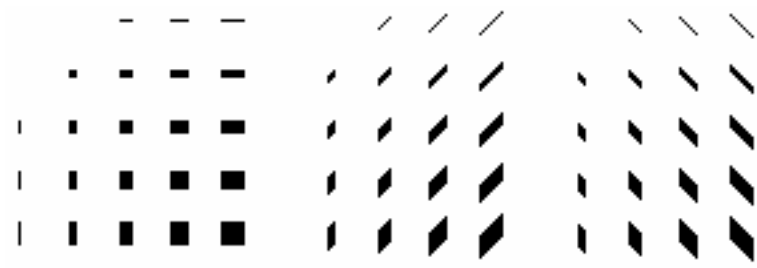

Figure 2 - Smoothing masks

Note that all the three levels are used, which allows the filter selection process to search masks with several frequency bandwidths, i.e., the filters applied the first level decomposition have the highest bandwidth.

In the second step the predefined set of smoothing filters is applied to each wavelet sub-band. This set includes square and rectangular windows, from $3 \times 3$ to $13 \times 13$ pixels, as well as 45 and 135 degrees rotated windows. Figure 2 shows these smoothing filters, only up to 9x9 and some small masks have been removed.

The third step selects the most generic masks; these are the masks that can detect most of the defects. The selection follows a sequential forward selection, successively adding the mask that detects the highest number of defects that are not detected by the already selected masks. To avoid too much filter specialisation, filters that detect a small number of defects are not considered for selection.

\section{RESULTS}

The proposed methodology has been applied to leather inspection. Each image sample was acquired with a resolution of 5.5 pixels per millimetre, achieving images with 2048x2048 pixels, which are processed to have an average constant intensity and contrast. Tests were performed on 80 images, containing 129 defects. Only the largest 200 regions on each image were analysed (areas that have high/low intensity and contrast), processing a total of 16000 regions. All the tests were performed using the Haar Wavelet.

The first test uses the same set for training and test. Its main goal is to test the ability of the proposed masks to separate normal from defect areas. This test gives an idea of the optimal detection rate that can be achieved with this type of filters. Figure 3 plots the number of defects detected as a functions of the number of masks used.

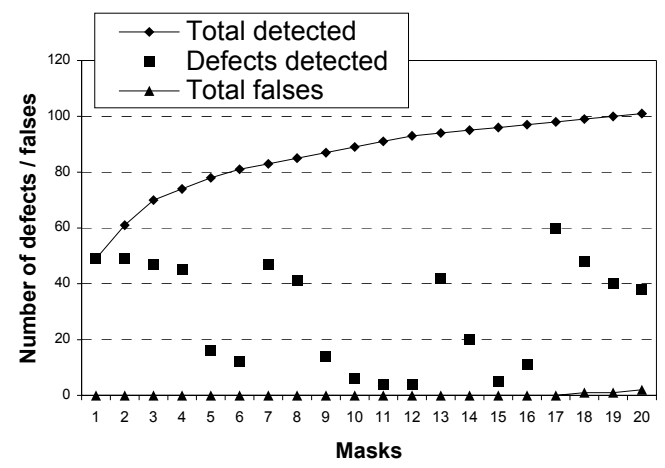

Figure 3 - Number of defects detected as a function of the number of masks 
TABLE I. FIRST TEN SELECTED MASKS

\begin{tabular}{|c|c|c|c|c|}
\hline $\mathbf{N}$ & Mask & Det. & $\Delta \mathbf{J}$ & $\mathbf{T}$ \\
\hline 1 & Q0 7x7 $0^{\circ}$ & 49 & 49 & 6.56 \\
\hline 2 & Q4 13x5 $0^{\circ}$ & 49 & 12 & 5.37 \\
\hline 3 & Q4 9x13 $0^{\circ}$ & 47 & 9 & 7.48 \\
\hline 4 & Q4 5x745 & 45 & 4 & 6.95 \\
\hline 5 & Q5 5x7 135 & 16 & 4 & 5.67 \\
\hline 6 & Q6 7x1145 & 12 & 3 & 6.33 \\
\hline 7 & Q4 5x3 $0^{\circ}$ & 47 & 2 & 5.50 \\
\hline 8 & Q0 7x5 135 & 41 & 2 & 6.14 \\
\hline 9 & Q8 13x9 $135^{\circ}$ & 14 & 2 & 5.61 \\
\hline 10 & Q5 $13 \times 30^{\circ}$ & 6 & 2 & 4.45 \\
\hline
\end{tabular}

The first 18 selected masks can detect 101 defects, $78 \%$ of the total, without introducing any false positive. Using more masks introduces false positives. The figure also includes the number of defects detected by each mask (Defects detected) and the total number of false. A total of 48 masks can detect all defects; however, the last 6 masks introduce a high number of false (from 10 and 50 false). These results indicate that the proposed methodology can effectively detect most leather defects.

Table I shows the first 10 masks selected, the number of defects detected (Det.), the additional defects detected $(\Delta \mathrm{J})$ and the threshold limit used for the classifier (T). When the output of one of the filters exceeds this value the region is considered a defect. The threshold limit is set to a value near of the first false positive. Figure 4 shows how the masks from this table are distributed in the wavelet sub-band decomposition. These 10 masks can detect 89 defects, $69 \%$ of the total.

Figure 5 illustrates inspection results. The defect in the first and in the second image are detected by mask "Q5 5x7 135", (mask number 5). The image on the right presents the results of this mask, before the classifier is applied. Several masks can detect the defect in the third image; the figure presents the results of mask "Q4 9x13 0".

Figure 6 presents some of the defects not detected by the first 20 masks selected. To detect these defects, using the proposed methodology, a high number of false is introduced.

The second experiment evaluates the generality of the selected masks, by using a different set of samples for training and for inspection. Half of the samples, randomly selected, were used to obtain the optimised filters (55 defects) and the other half were used in the inspection process ( 74 defects).
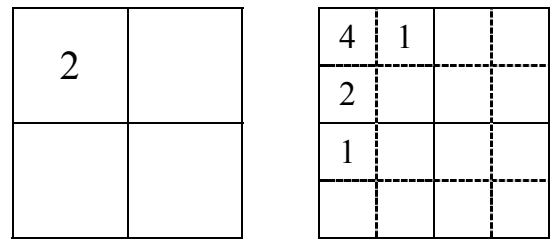

Figure 4 - Wavelet sub-bands of each selected filter
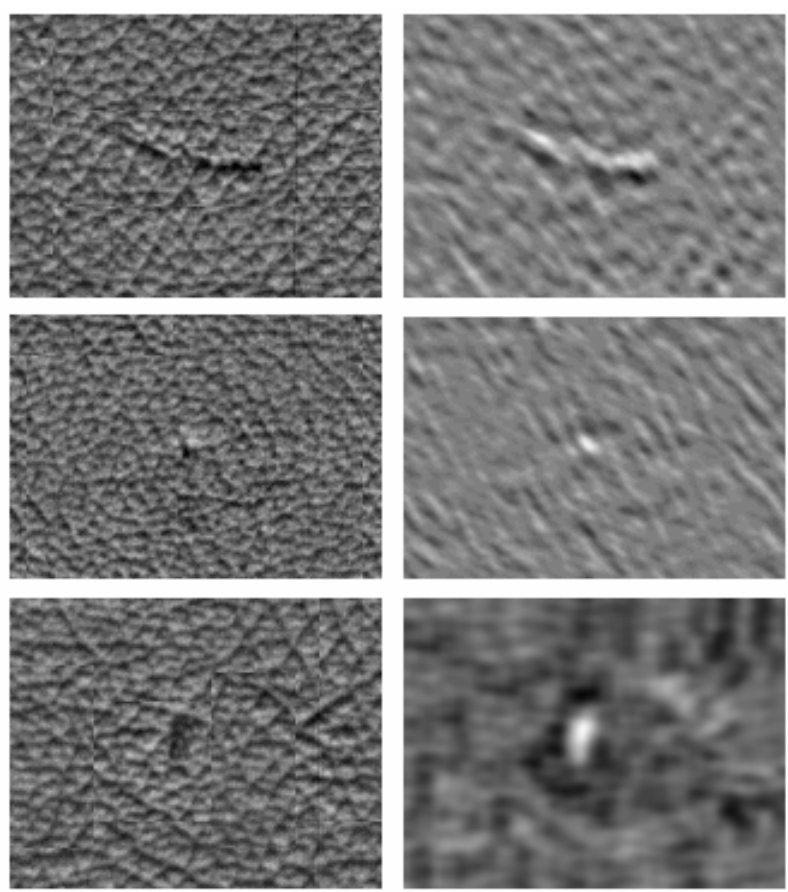

Figure 5 - Optimised masks results

Figure 7 presents the percentage of detected defects as a function of the number of masks selected. The same figure also presents the percentage of false positives.

These results show that the masks selected in the training process are generic: they were able to detect $60 \%$ of defects when using only 8 masks. Using a higher number of masks rapidly increases the number of false positives; as such, the number of masks should be relatively low. However, most of false positives obtained with a larger number of masks may be due to the small set used for training, since there were 55 defects, which results in an average of less than 7 per mask, when using more than 8 masks.
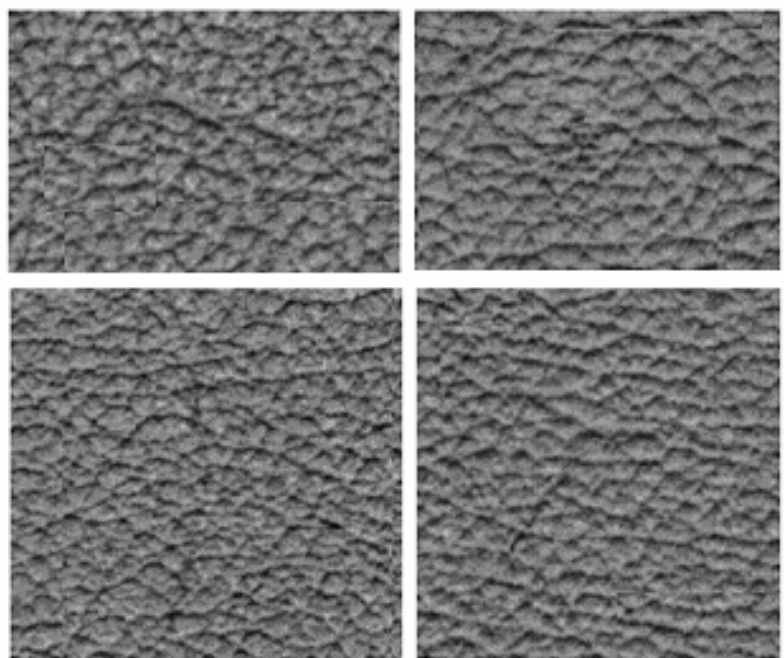

Figure 6 - Some defects not detected by the first 20 masks 


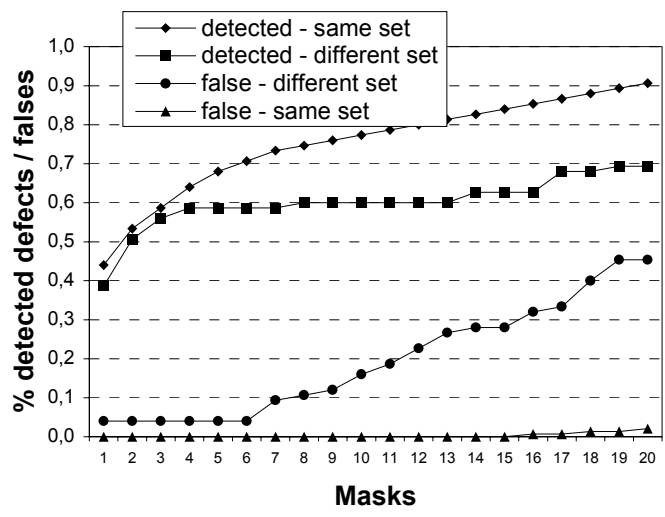

Figure 7 - Number of defects detected, when using the same and a different set for training and for test

Note that many leather defects are very difficult to detect manually; it is estimated that a highly trained human operator can detect about $60 \%$ to $70 \%$ of defects. Also, human operators can work only a small amount of time and different operators usually detect different defects.

\section{CONCLUSIONS}

This article described a new methodology to detect defects on textured materials. The methodology is based on a recursive power spectrum partition, provided by a wavelet packet transform, and on a set of filters applied to each sub-band. Each filter size and shape and wavelet sub-band is tuned detected a type of texture defects.
The presented results on leather inspection showed that these masks can detect most of the defects and that these masks are generic, since they achieved an acceptable detection rate when tested on new set of samples. Also, these results should receive a high improvement by using a larger data set for training.

Current work includes experiments with other wavelet transforms, to select the best wavelet bases, which should improve the recognition rate but also will increase the processing time.

\section{REFERENCES}

[1] T. Randen, J. Husoy, "Filtering for Texture Classification: A Comparative Study", IEEE Transactions on Pattern Analysis and Machine Intelligence, v. 21 n. 4, April 1999.

[2] A. Jain, F. Farrokhnia, "Unsupervised Texture Segmentation Using Gabor Filters", IEEE International Conference on Systems, Man and Cybernetics, November 1990.

[3] A. Bodnarova, M. Bennamoun, J. Latham, "A Constrained Minimisation Approach to Optimise Gabor Filters for Detecting Flaws in Woven Textiles", IEEE International Conference on Acoustics, Speech, and Signal Processing, June 2000.

[4] A. Kumar, G. Pang, "Defect Detection in Textured Materials Using Gabor Filters", IEEE Transactions on Industry Applications, v. 38 n.2, March/April 2002.

[5] S. Mallat, "A Theory for Multiresolution Signal Decomposition: The Wavelet Representation", IEEE Trans. Pattern Analysis and Machine Intelligence, v. 11 n. 7, July 1989.

[6] A. Laine, J. Fan, "Texture Classification by Wavelet Packet Signatures", IEEE Transactions on Pattern Analysis and Machine Intelligence, v. $15 \mathrm{n}$. 11, November 1993. 